\title{
PREDICCIÓN DE LA POTENCIALIDAD DE LOS BOSQUES ESCLERÓFILOS ESPAÑOLES MEDIANTE REDES NEURONALES ARTIFICIALES
}

\author{
M. Benito Garzón ${ }^{1}$ J. Maldonado Ruiz², R. Sánchez de Dios ${ }^{1}$ y H. Sainz Ollero ${ }^{1}$
}

\begin{abstract}
RESUMEN
Encinares y alcornocales son dos de las formaciones esclerófilas más importantes de la Iberia mediterránea. Para conocer cual es su potencialidad en el territorio español se ha empleado un modelo generado mediante redes neuronales artificiales con un algoritmo de retropropagación de errores que conduce la información siempre hacia delante. Las variables bioclimáticas empleadas como predictores son: altitud, continentalidad, insolación, precipitación total, temperatura media anual, temperatura media de las mínimas del mes más frío y temperatura media de las máximas del mes más cálido, con una resolución de $10 \mathrm{~km}$. Las redes neuronales se perfilan como una herramienta de gran poder predictivo. Se aprecian patrones de respuesta diferente para las formaciones estudiadas. Mientras que para la encina se simula un área potencial continua y extensa, para el alcornoque se obtiene un área fragmentada y restringida, que se ajusta bastante a su presencia actual. La principal discrepancia del modelo presentado con esquemas de vegetación potencial anteriores radica en la ausencia de encinares y alcornocales en zonas térmicas del Levante y sur peninsular.
\end{abstract}

Palabras clave: vegetación potencial, redes neuronales artificiales, Quercus ilex, Quercus suber, encina, alcornoque

\begin{abstract}
Predicting spanish sclerophyllous forests potentiality using artificial neural networks

Holm oak and cork oak forests are between the most important sclerophyllous formations in the Mediterranean Iberia. In order to study their potentiality, an artificial neural network model, with a feedforward BP algorithm, has been applied. The elevation, continentality, insolation, annual rainfall, annual mean temperature, mean temperature of the coldest month and mean temperature of the warmest month are the used bioclimatic variables with a $10 \mathrm{~km}$ resolution. The neural networks seem a highly predictive powerful tool. Different patterns in the response of the studied forests have been shown. The holm oak presents a continuous and wide potential simulate range. Meanwhile the cork oak potential area is fragmented and restricted, in accordance with its actual distribution area. The lack of both forests in the eastern and southern warm zones of Iberian Peninsula is the main discrepancy with previous potential vegetation proposals.
\end{abstract}

Keywords: potential vegetation, artificial neural networks, Quercus ilex, Quercus suber, Holm Oak, Cork Oak.

\footnotetext{
Departamento de Biología (Botánica), UAM.

Unidad de Botánica, Departamento de Silvopascicultura, UPM.
} 


\section{Introducción}

La profunda transformación que han sufrido los paisajes mediterráneos ha provocado, desde hace años, interpretaciones más o menos arriesgadas en términos de potencialidad de las especies vegetales. La multicausalidad de los procesos ecológicos ha llevado al intento de modelización de patrones de distribución de las especies mediante diversas técnicas estadísticas (Terradas, 2001). En los últimos años se están empleando técnicas computacionales alternativas conocidas como "soft computing" que comprenden Redes Neuronales Artificiales (RNA), Lógica Borrosa, Algoritmos Genéticos, etc. Básicamente, difieren de otras técnicas en que son capaces de "aprender" en un sentido biológico: en su origen intentaban emular la forma de aprendizaje humano, ahora se podrían incluir dentro de las técnicas de "aprendizaje automático". Las RNA reconocen patrones en los datos de entrada que reproducen mediante un sistema de pesos ajustables entre las neuronas de diferentes capas, proporcionando una respuesta que asemeja esos patrones.

Recientemente, se han empleado RNA para simular dinámicas caóticas y complejas, como pueden ser el clima (Lennon \& Turner, 1995; Lee et al., 1998; Bryan \& Adams, 2001; Pérez-Llera, 2002), o las mareas rojas (Lee et al., 2003), que de otra forma serían difícilmente predecibles.

La estimación de la distribución potencial de las especies se ha realizado en este trabajo mediante RNA, esta técnica se ha elegido frente a otras debido a su gran capacidad de procesamiento de relaciones no lineales complejas y a que no necesita asumir normalidad en los datos de partida.

Sin embargo, su mayor desventaja es que no se puede conocer con exactitud el aporte de cada uno de los predictores al modelo, sino que obtenemos un resultado global que incluye a todos los predictores. Existen otras técnicas de modelización que si permiten conocer en que medida contribuyen los predictores en el resultado final (y que también permiten trabajar con relaciones no lineales). Han sido ampliamente estudiadas por estadistas y entre las más conocidas se encuentran las siguientes:

- Regresiones logarítmicas y modelos logit, diseñados a partir de los modelos de regresiones lineares para poder trabajar con relaciones no lineares mediante transformaciones logarítmicas (Augustin et al., 2001)

- Generalized Additive Models (GAM) (Guisan et $a l, 2002)$ se podrían utilizar con el mismo objetivo de modelizar que las RNA, si bien los prime- ros resultan más complejos en su construcción y no permiten interacciones entre las variables de entrada, no son capaces de modelizar una función del tipo $\mathrm{X}_{1} \mathrm{X}_{2}$ (Bishop, 1995).

- Multivariate Adaptative Regression Splines (MARS) (Friedman, 1991) que, a diferencia del anterior, sí permiten las interacciones entre las variables predictoras.

- Árboles de clasificación y regresión (CART) basados en la separación binomial del hiperespacio. Este tipo de análisis ha sido ampliamente utilizado con el mismo objetivo que en este trabajo (Miller \& Franklin, 2002).

El estudio de los patrones que determinan la distribución de la vegetación es claramente complejo, y además de verse condicionado por variables climáticas y edáficas (clímax en sentido de Clements), se ve afectado por factores históricos, dispersión y colonización, competencia entre especies, etc. Aunque en este trabajo se desarrolla un modelo exclusivamente climático, los factores históricos quedan implícitos de forma indirecta en la distribución actual de las especies, que es asimismo incluida en el modelo como predictor.

La predicción de hábitat de las especies tomadas individualmente, o estudio de áreas potenciales, se ha basado hasta hace relativamente poco en ideas intuitivas. Sin embargo, en los últimos años se está avanzando en este campo gracias a las buenas bases de datos cartográficas y a la mejora de las técnicas predictivas. Algunos ejemplos se observan en las nuevas tendencias centroeuropeas y norteamericanas en la predicción de hábitat con la asunción previa de cambio climático (Huntley et al., 1995; Sykes et al., 1996; Iverson et al., 1998; Iverson et al., 1999; Parmesan et al., 1999; Pearson et al. 2000; Berry et al., 2001). Los modelos realizados en los últimos años intentan probar, a su vez, nuevas metodologías, por ejemplo, un análisis de componentes principales usado para la predicción de hábitat (Robertson et al., 2001), introduciendo algunas variantes en el ENFA ("Ecological-Niche Factor Analysis") (Hirzel et al., 2001), donde sólo se usan las presencias para predecir las distribuciones potenciales (Hirzel et al, 2002); uso de los datos fitosociológicos bibliográficos en la predicción de la vegetación bajo los efectos del cambio climático (Duckworth et al., 2000); árboles de clasificación para predecir las distribuciones de las especies (Vayssières et al., 2000), y otros que insertan modificaciones en métodos predictivos clásicos, como el realizado mediante regresiones logísticas por Augustin et al., (2001), entre otros. De 
forma paralela aparecen trabajos cuyo objetivo principal es la comparación entre diferentes técnicas predictivas (Guisan et al., 1999; Paruelo \& Tomasel, 1997; Hilbert \& Ostendorf, 2001). Resulta difícil, a priori, saber que técnica predictiva será la más apropiada para los datos de que se dispone. Sin embargo, empiezan a aparecer trabajos que intentan recopilar información que pueda ser de ayuda para la creación del modelo que necesitemos según los datos de partida y los objetivos que se persigan (Guisan \& Zimmermman, 2000, Seoane y Bustamante, 2001).

A pesar del auge de la modelización, y las diferentes metodologías, aún no se ha llegado a un consenso sobre las herramientas a utilizar para cada caso particular. En este trabajo se pretende aplicar la predicción mediante RNA en España peninsular y Baleares. Para ello, se han utilizado dos especies emblemáticas peninsulares: la encina $\mathrm{y}$ el alcornoque.

\section{DiSTRIBUCIÓN Y ECOLOGÍA DE LOS ENCINARES Y ALCORNOCALES ESPAÑOLES}

Encinares y alcornocales son los bosques de frondosas esclerófilas (hojas planas, perennes y coriáceas) más característicos de la Iberia seca. Los encinares son los bosques más típicos de España, donde alcanzan la mayor extensión europea, mientras que los alcornocales son más abundantes en Portugal. La encina (Quercus ilex L.) y el alcornoque (Quercus suber L.) son taxones antiguos (Terciarios) que se encuentran bien representados en el Mediterráneo, especialmente en su mitad occidental (Iberia y el Magreb).

Mientras el alcornoque parece una especie relativamente estable en términos evolutivos, la encina exhibe una gran variabilidad morfológica y ambiental lo cual da lugar a que se reconozcan dos formas o subespecies. Quercus ilex subsp. ilex, de hojas lauroides y bellotas amargas, que parece ser la más antigua, al estar adaptada a un clima mediterráneo-templado relativamente húmedo; y Quercus ilex subsp. ballota (Desf.) Samp. (= Q. rotundifolia Lam.) de hojas pequeñas redondeadas, frecuentemente espinosas, y bellotas dulces. Esta última resiste bien la continentalidad, soportando fuertes heladas invernales. En España, para referirse a las dos encinas, resulta útil emplear, atendiendo a las áreas en que predominan, la denominación catalana de "alsina" para la primera y la castellana de "carrasca" para la segunda.

La carrasca, la subespecie más extendida, puede llegar a soportar temperaturas cercanas a los $-20^{\circ} \mathrm{C}$ sin sufrir lesiones y es más frugal y xerófila; por ello se piensa que puede haber cobrado importancia paisajística en relación con la evolución del clima en el Cuaternario. Habría desarrollado la resistencia al frío en los periodos glaciares extendiéndose sobre todo en los últimos milenios coincidiendo con el impacto antrópico sobre otros bosques más exigentes, especialmente quejigares, alcornocales y melojares. Este proceso también estaría directamente influido por el hombre debido al interés de sus bellotas ("frutalización", González Bernáldez, 1992).

En la península Ibérica se concentra la mayor superficie de alcornocales dado que entre España y Portugal reúnen cerca de un millón de hectáreas, lo que representa aproximadamente la mitad de su área de distribución mundial (Montoya, 1988; Díaz-Fernández et al., 1995). La especie que da nombre a estas formaciones, Quercus suber L., es un taxon estenoico, termófilo y estrictamente silicícola. Bastante más exigente en humedad que la carrasca y algo menos que la alsina. Los países en los que tienen más importancia los alcornocales son Portugal, España, Argelia, Marruecos, Francia, Túnez e Italia. Sus formaciones están presentes en casi todas las islas del mediterráneo occidental: Cerdeña, Córcega, Sicilia, Menorca, y también se presentan de forma puntual en algunos países del mediterráneo oriental: Croacia, Albania y Grecia.

$\mathrm{Su}$ distribución presenta numerosas disyunciones, siendo frecuente la presencia de poblaciones, rodales e incluso pies aislados que se interpretan habitualmente en relación con su antigüedad y regresión reciente. Esa fragmentación de sus poblaciones, debida tanto a razones antrópicas como paleogeográficas, aproxima esta especie a otros taxones paleomediterráneos como la alsina, el loro (Prunus lusitanica L.) o el laurel (Laurus nobilis L.) y la aleja de la carrasca. Los alcornocales más extensos se localizan en el suroeste ibérico y el noroeste de Marruecos.

\section{POTENCIALIDAD EN EL TERRITORIO ESPAÑOL}

En general, se tiene la idea de que, en la mayor parte de la Iberia mediterránea, la "clímax" correspondería a encinares y alcornocales y que en el pasado bosques de estas características cubrirían casi por completo el territorio (el mito de la "ardilla viajera" atribuido a Plinio). Se estima que entre el 60 y el $80 \%$ de la superficie ibérica (unos 30 millones de hectáreas) correspondería a este tipo de bosques (Font Quer, 1954; López González, 2001). De ellos solo queda alrededor de un $18 \%$, unos 5,5 millones (Maldonado et al., 2002). Las manifestaciones actuales de estas comunidades 


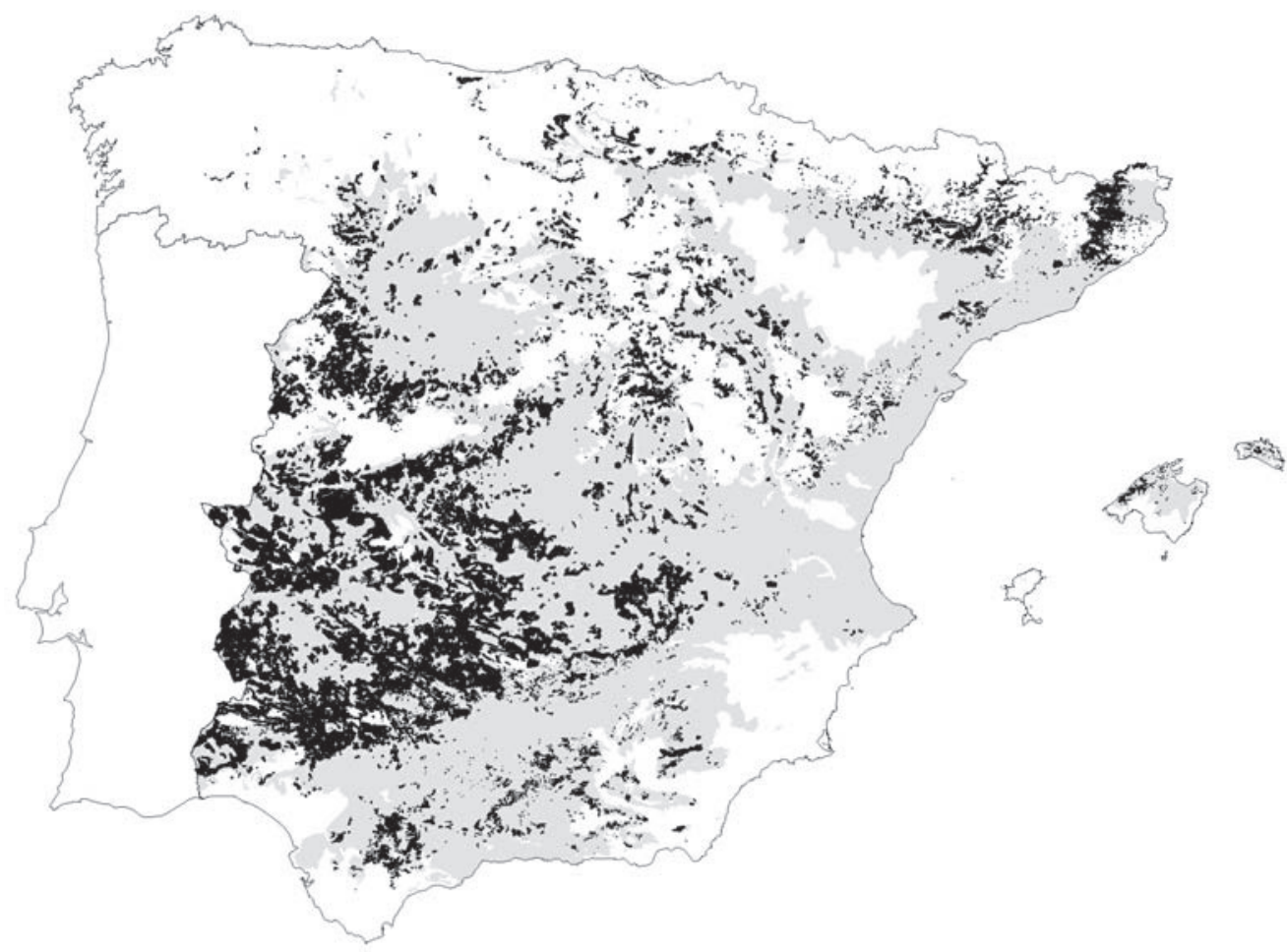

Fig. 1.- Distribución, en negro, de los encinares españoles según el mapa forestal de Ceballos (1966) y área potencial, en gris, según Rivas-Martínez (1987).

Fig. 1.- Actual distribution of spanish holm oak forests (solid) after Ceballos (1966) and potential area (grey) after RivasMartínez (1987).

constituyen restos del bosque primigenio, pero presentan características muy distintas a las de las formaciones originales, pues es notoria la incesante actuación humana sobre el paisaje ibérico, que se prolonga desde tiempos prehistóricos. Pero, ¿está comprobada dicha potencialidad en toda la Iberia mediterránea?

Frente a la intensidad de la alteración humana sobre el medio y la imposibilidad de conocer con exactitud la distribución en el pasado de las distintas comunidades vegetales, han cobrado bastante éxito los esquemas de "vegetación potencial". Tradicionalmente en España, a partir de 1950 , la interpretación de la vegetación potencial se ha realizado desde la óptica de la metodología fitosociológica, culminando con la publicación del Mapa de Series de Vegetación de España (Rivas-Martínez, 1987). Éste ha alcanzando gran difusión y se presenta como una útil herramienta para la planificación y la restauración forestal o paisajística.
En este sentido, Maldonado et al. (2002) han aportado algunos datos acerca de la correlación existente entre la distribución real de encinares y alcornocales y sus áreas potenciales en el mapa de series (Rivas-Martínez, 1987). La potencialidad de los encinares es bastante más virtual o especulativa que la de los alcornocales. En el caso de los primeros el área real supone el $17 \%$ de una superficie potencial que representa el $57,8 \%$ de la España peninsular (fig. 1). A pesar de su gran extensión resulta alrededor de un $20 \%$ menor que el área propuesta por Font Quer (1954). El territorio potencial se extiende de forma casi continua por la mayor parte de la región mediterránea (pisos termo, meso y supramediterráneos) con excepción de los macizos montañosos, las zonas semiáridas y las ocupadas por alcornocales. Señalan también, que algunas series pudieran estar sobredimensionadas, especialmente en el piso termomediterráneo (valle del Guadalquivir y litoral levantino) y en la provincia castellano-maestrazgo-manchega. 


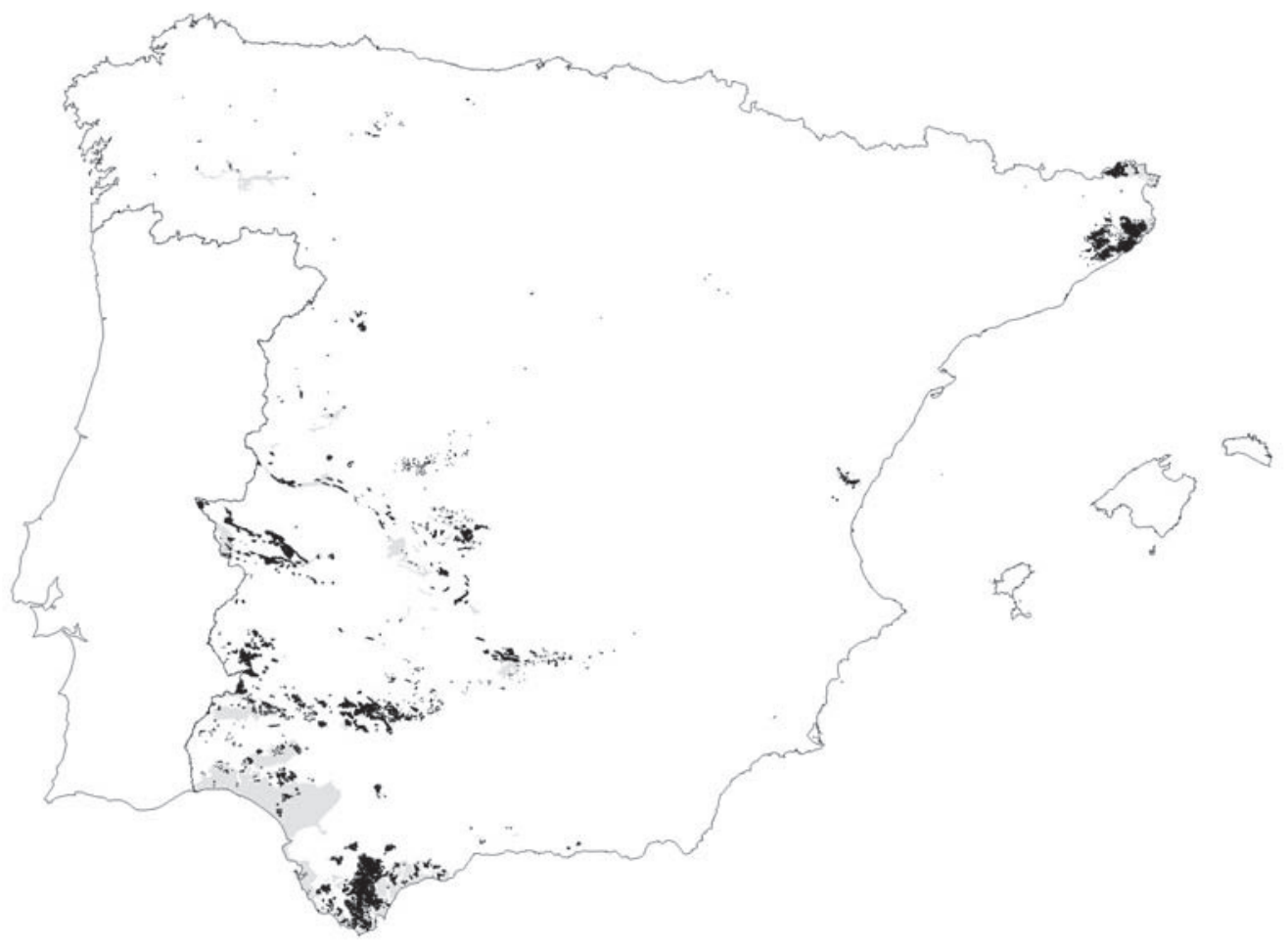

Fig. 2.- Distribución, en negro, de los alcornocales españoles según el mapa forestal de Ceballos (1966) y área potencial, en gris, según Rivas-Martínez (1987).

Fig. 2.- Actual distribution of spanish cork oak forests (solid) after Ceballos (1966) and potential area (grey) after RivasMartínez (1987).

La delimitación de las series potenciales de los alcornocales ha sido mucho más fiel a la distribución actual de estos bosques (fig. 2), salvo en el litoral gaditano-onubo-algarviense. No se han considerado las abundantes localidades aisladas de la especie (Liébana, litoral galaico y cantábrico, alto Ebro, Sierra de la Virgen, Hoyo de Manzanares, etc.) que a juicio de muchos autores ponen de manifiesto una distribución pasada mucho mayor que la actual (Font Quer, 1954; Ceballos y Ruiz de la Torre, 1971; Montoya, 1988; Costa Tenorio et al., 1997). El área potencial representa el 3,3\% de la España peninsular y hay constancia de estas formaciones en un $31 \%$ de esa superficie $(60 \%$ excluyendo las marismas de Huelva y Sevilla).

Hasta ahora casi no existen otros trabajos dedicados a la modelización de la vegetación sensu stricto, aunque en los últimos años se detecta una inquietud en este sentido integrada mayoritariamente por aportaciones inéditas que modelizan las distribuciones vegetales mediante regresiones (Ninyerola, 2000; Martínez, 1997).

El empleo de Sistemas de Información Geográfica y de herramientas predictivas como las redes neuronales artificiales abren nuevas posibilidades en el campo de los modelos geobotánicos de vegetación potencial. Con estas nuevas metodologías de modelización se puede abordar de una forma rigurosa y contrastable la potencialidad de encinares y alcornocales.

\section{Metodología}

El cálculo del área de ocupación potencial de los encinares y alcornocales en la España peninsular y Baleares se ha realizado partiendo de las distribuciones del Mapa Forestal de Ceballos (1966) (figs. 1 y 2) a escala 1:400. 000. En este mapa no se separan las dos subespecies de Quercus ilex, por lo que la potencialidad estimada en este trabajo 
corresponde al conjunto de la especie. Se han utilizado para la predicción un total de siete variables bioclimáticas: altitud (derivada del modelo digital de elevaciones GTOPO30, de aproximadamente 1 $\mathrm{km}$ de resolución), continentalidad (índice de Gorezynski), insolación, precipitación total, temperatura media anual, temperatura media de las mínimas del mes más frío y temperatura media de las máximas del mes más cálido.

Los datos climáticos proceden de más de 2600 estaciones del Instituto Meteorológico Nacional que abarcan el periodo comprendido entre $1940 \mathrm{y}$ 1989. A partir de las coordenadas geográficas de las estaciones meteorológicas, cada variable climática se ha interpolado a todo el territorio a una resolución de $10 \mathrm{~km}$. Se ha elegido para la interpolación el método de mínima curvatura (Briggs, citado en Mitásová \& Mitas, 1993). Este método presenta ventajas frente a otros utilizados en interpolaciones similares (Sánchez Palomares et al., 1999), puesto que ajusta a la variable una ecuación con la que se describe una superficie ondulada. La variante empleada en este estudio se puede considerar equivalente a una lámina fina, elástica y lineal que pasase a través de cada valor con una mínima curvatura. De esta forma, se puede lograr un gradiente en zonas de "meseta" en la superficie de interpolación, como son las áreas de montaña o las zonas bajas, donde apenas existen estaciones meteorológicas.

Dentro de las RNA existen una amplia gama que se han venido utilizando en diseños diversos, si bien el perceptrón multicapa resulta el más polivalente y sin duda, el más citado en la literatura y sobre el que se tiene más conocimiento. Así mismo, es fácil de encontrar buenas implementaciones gratuitas y abiertas, como el simulador utilizado en este trabajo, SNNS (http://www-ra. informatik. uni-tuebingen. de/SNNS/).

Los perceptrones multicapa forman una familia de aproximación funcional para cualquier función continua y derivable, por lo que, en principio, resultan un buen método de modelización: se busca un modelo que se intuye que existe. El aprendizaje realizado por los perceptrones multicapa es un problema de minimización de una función de error mediante el cálculo del gradiente. El método que se utilice para esta minimización por gradiente resulta clave para que el modelo converja a un buen resultado (LeCun et al, 1998).

La unidad basica de cómputo en los perceptrones multicapa (y las RNA) son los nodos o neuronas, que definen una transformación global de las entradas según una función de activación. La fun- ción de activación utilizada en este trabajo ha sido la siguiente:

$$
\mathrm{a}_{\mathrm{j}}=\sum \mathrm{w}_{\mathrm{ij}} \sigma(\mathrm{x}) \text { función de activación }
$$

$\sigma(\mathrm{x})=\mathrm{x}$ activación en las capas de entrada

$\sigma(\mathrm{x})=1 /(1+\exp (-\mathrm{a}))$ activación sigmoidal en las capas ocultas que limita los outputs entre 0 y 1 .

El poder de cálculo de las redes de neuronas se asienta básicamente en el procesamiento en paralelo, la adaptabilidad al entorno y memoria distribuida.

La estructura básica del perceptrón multicapa diseñado para este trabajo consta de una capa de entrada con 7 nodos, otra oculta con 11 y otra de salida con 1. En las conexiones y los pesos entre las conexiones es donde radican las diferencias entre las redes de neuronas (Goh, 1995). Es importante una buena selección de la arquitectura neuronal en el modelo que se quiera desarrollar. Así, si el número de neuronas es demasiado elevado se corre el riesgo del sobreajuste, que impide la buena generalización. Si, al contrario, se elige un diseño con pocas neuronas el algoritmo no llega a converger, y el modelo presenta un mal ajuste. En general, este diseño de la arquitectura suele consistir en ensayo y error hasta dar con una arquitectura que satisfaga las necesidades del modelo, aunque existen algunas aproximaciones matemáticas (Baum \& Haussler, 1989).

La RNA que se ha utilizado ha sido el perceptrón multicapa (MLP), ampliamente utilizado desde que Hornik et al. (1989) demostraron que podía ser entrenado por cualquier función medible suavizada (Gardner \& Dorling, 1998). En este caso se ha utilizado para entrenar a la red el algoritmo de retropropagación de errores (BP):

Los perceptrones multicapa basan su aprendizaje en una minimización de la función de error respecto a los pesos y sesgo.

$$
E^{k}=1 / 2 \sum\left(y_{k}-t_{k}\right)^{2} \text { función de error (Bishop, 1995) }
$$

Esta minimización de la función de error se puede llevar a cabo por diferentes algoritmos, uno de los más utilizados debido a su solidez es el BP. Este algoritmo evalúa las derivadas de la función de error anterior en dos pasos secuenciales, uno primero de evaluación respecto a los pesos y otro de ajuste a esos pesos.

Aprendizaje y generalización son, en el diseño de redes neuronales, un compromiso entre sesgo y varianza, para ello se debe controlar la complejidad efectiva del modelo (Bishop, 1995). Existen varias 
formas de hacerlo, si bien una de las más prácticas es parar el aprendizaje en el momento óptimo mediante la técnica que aquí se ha empleado de validación cruzada.

Los datos de partida requieren un procesamiento antes de utilizar el algoritmo BP, debido a que se ha utilizado una función de activación sigmoidea. La función de normalización empleada homogeniza los valores entre 0 y 1 (Goh, 1995). La conexión inicial de los pesos entre nodos se seleccionó al azar entre los valores de 2. 0 y -2 . 0 (valores de conexión de pesos recomendados: pequeños, positivos y negativos, Martín y Sanz, 1997) y el parámetro de aprendizaje fue establecido en 2. La validación del modelo se realizó cada 100 ciclos. Con esto se puede identificar cuando la red comienza a sobregeneralizar y el aprendizaje puede ser parado en el punto donde se obtenga el mínimo error, así se asegura que la red neuronal funciona reduciendo al mínimo la diferencia entre los datos observados y los predichos (validación cruzada).

Para un correcto funcionamiento de una red entrenada con este algoritmo los datos deben ser divididos en tres subconjuntos aleatorios (cada uno supone un tercio del total de los datos), uno para entrenar la red, otro para probar el funcionamiento de este adiestramiento y un tercero para validar el modelo independientemente, mediante el coeficiente de correlación de Pearson. La red se entrena $\mathrm{y}$ valida al mismo tiempo deteniéndose en el punto óptimo, en un procedimiento denominado validación cruzada o cross-validation (Martín y Sanz, 1997; Evans, 1998). Cuando la red alcanza el mínimo error cuadrado en el aprendizaje, alcanza el máximo poder de generalización en la predicción, y es el entrenamiento que se debe seleccionar para la predicción, evitando caer en mínimos locales. Este patrón de calibrado del modelo solo puede ser utilizado cuando se dispone de grandes cantidades de datos, como en este caso, que, a la resolución utilizada asciende a 6783 celdas para el conjunto de la península. Es decir, se entrena la red con 2261 datos.

La red genera mapas de probabilidades de aparición de las especies desde 0 hasta 1 (gracias a la función de activación sigmoidal utilizada). Para seleccionar las probabilidades que más se ajustan a la distribución inicial se ha utilizado el estadístico de similitud kappa (Carletta, 1996). Valores por debajo de 0,4 representan poco grado de semejanza, entre 0,4 y 0,55 un grado de semejanza aceptable, entre 0,55 y 0,7 bueno, de 0,7 a 0,85 muy bueno, y mayor de 0,85 excelente (Monserud, 1990). Aunque se debe tener siempre en cuenta que las predicciones en ecología no suelen ser muy potentes, estando comprendidas en un rango de predictibilidad de entre 20\% y 70\% (Kirkby, 2000).

\section{Resultados}

El objetivo final de los modelos es la generalización, que también puede definirse como la capacidad de predicción del modelo. En RNA la mejor predicción no viene dada por el mínimo error en el conjunto de aprendizaje, que suele terminar en sobreajuste de la red a los datos de partida, sino por la capacidad de generalización. En RNA los problemas de generalización tienen varias formas de abordarse, una de las más utilizadas es para el entrenamiento del conjunto de datos en el momento óptimo, lo cual se consigue mediante validación cruzada, tal y como se explica en el apartado metodológico de este trabajo.

Con los mapas de probabilidades obtenidos como resultado de este proceso se midió el grado de similitud a los mapas actuales de vegetación de las especies mediante el estadísco de similitud kappa, escogiendo entre todos los resultados el que tuviera mayor grado de similitud con el mapa actual real de vegetación.

En los mapas se representan tanto los mapas de probabilidades seleccionados con validación cruzada como el punto de corte seleccionado mediante el estadístico de similitud.

\section{ENCINARES}

En la figura 3 se presenta la probabilidad de aparición de los encinares en la España peninsular y las islas Baleares. En el recuadro inferior aparece el área resultante de aplicar el valor de corte basado en el estadístico kappa.

El área predicha para esta especie, según el modelo utilizado, está completamente ausente de la Iberia atlántica y de la alta montaña pirenaica. Se extiende de forma más o menos continua por todo el interior de la Iberia mediterránea con excepción de las partes más altas de los sistemas montañosos, el valle del Ebro, incluido su afluente el Jalón, y los tramos inferiores del Duero y el Guadiana. La probabilidad de presencia alcanza valores máximos en la submeseta sur, especialmente en La Mancha y La Alcarria, en Extremadura, y en el piedemonte de las sierras Prebéticas y Prepirenaicas. Sin embargo, no resulta favorable para esta especie una banda continua a lo largo del Levante que alcanza su máxima anchura en el sudeste y penetra hacia el interior por algunos de los valles más térmicos (Turia, Júcar, 


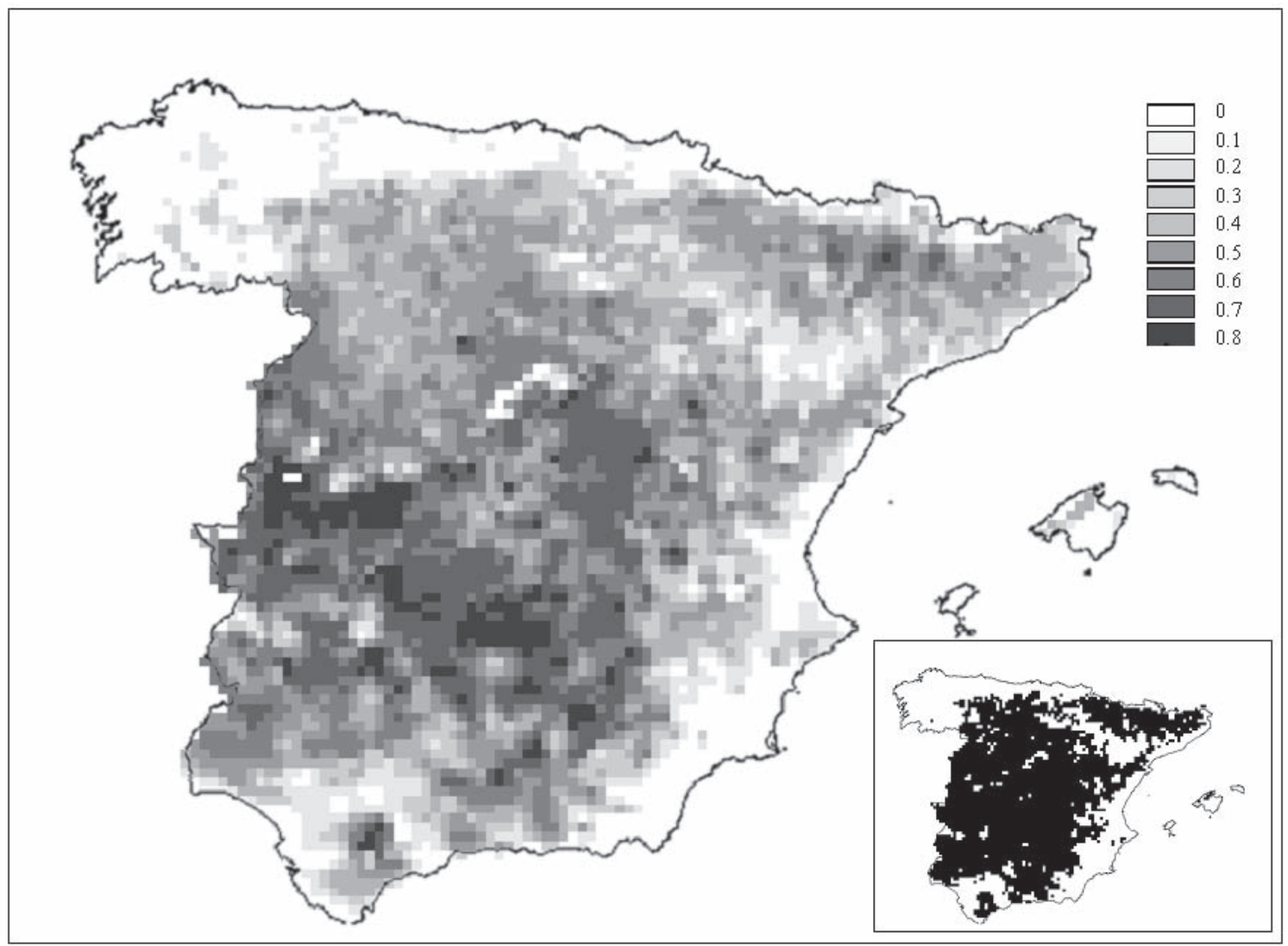

Fig. 3.- Probabilidad de aparición de la encina mediante redes neuronales artificiales. En el recuadro se muestra el nivel de corte para una probabilidad de 0.3 elegida según el estadístico kappa.

Fig. 3.- Predicted probability of holm oak using artificial neural networks. The small window shows a kappa cut-off threslhold of 0.3 .

Segura...). Tampoco aparecen como zonas potenciales el valle del Guadalquivir, el litoral gaditanoonubense y el valle del Guadalhorce.

Destaca también la presencia puntual, aunque a veces con probabilidades bajas en la sierra de la Tramontana y península de Artá, en Mallorca, en las sierras alicantinas, en los cañones gallegos del Sil y del Miño, junto a otros de la cornisa Cantábrica, y en los valles del Noguera Pallaresa y Segre, en el Pirineo central.

\section{AlCoRnOCALES}

La aplicación de redes neuronales a la distribución española de los alcornocales genera un área favorable potencial que recuerda bastante a la distribución actual de estos bosques. Como en el caso anterior, el recuadro inferior de la figura 4 representa el área más probable según el estadís- tico de similitud kappa. Está organizada en cuatro núcleos:

- uno catalán, con centro de dispersión en la comarca de la Selva

- otro gaditano-malagueño que reúne la Sierra del Aljibe y Sierra Bermeja

- un tercero extendido por la mitad occidental de Sierra Morena, desde Los Pedroches

- y el cuarto en la Sierra de San Pedro y otras elevaciones de la provincia de Cáceres. Además aparecen algunas cuadrículas aisladas en la Marina Alta de Alicante, en el Bajo Maestrazgo o en Mallorca, cerca de Pollensa.

El área predicha no recoge todos los puntos de la distribución de partida. Aunque confiere continuidad a algunas zonas, en líneas generales es algo más restringida. Llama la atención la ausencia en 


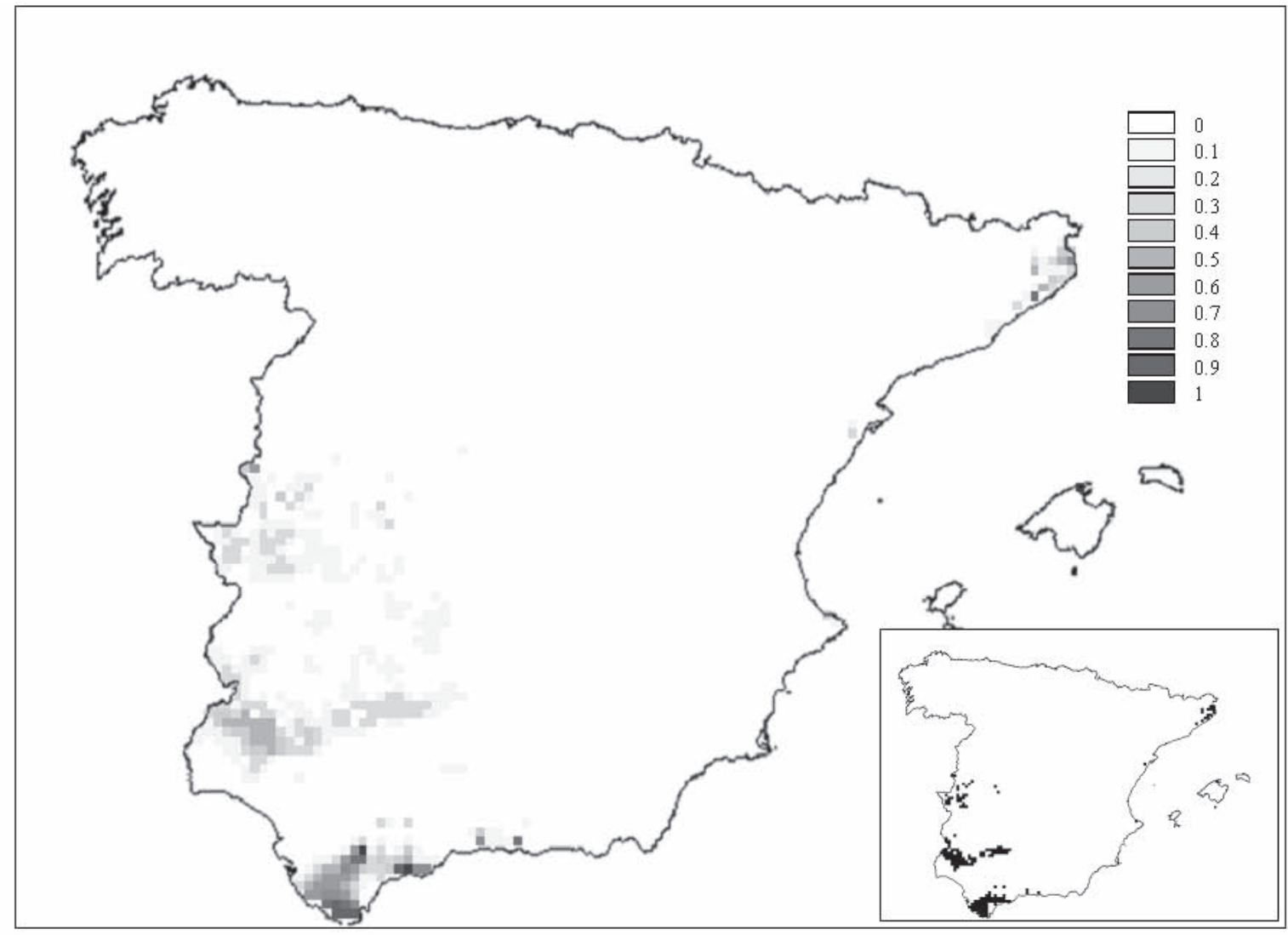

Fig. 4.- Probabilidad de aparición del alcornoque mediante redes neuronales artificiales. En el recuadro se muestra el nivel de corte para una probabilidad de 0.2 elegida según el estadístico kappa.

Fig. 4.- Predicted probability of cork oak using artificial neural networks. The small window shows a kappa cut-off threslhold of 0.2 .

Montes de Toledo o en el extremo oriental de Sierra Morena y en toda la submeseta norte. Tampoco aparecen los alcornocales castellonenses de la Sierra de Espadán.

\section{Discusión}

El modelo empleado predice la disponibilidad de hábitat de una especie, pero no la vegetación potencial en el sentido habitualmente empleado (Rivas-Martínez, 1987, pag. 171). En este tipo de modelos, la ausencia nos indica que en el territorio en cuestión no se dan las condiciones climáticas necesarias para que la especie pueda vivir. La presencia, por el contrario, aunque el clima sea favorable, puede estar limitada por otros factores (históricos, dinámicos, paleogeográficos...), por lo que la especie en cuestión puede no ser dominante en la actualidad.

La modelización de especies lleva implícita una serie de limitaciones que hay que tener en cuenta a la hora de interpretar los resultados. Algunas de ellas son inherentes a los datos de partida: las distribuciones de las especies y los predictores elegidos. Otras dependen de la escala de trabajo, variando si se trata de modelar macrotendencias, o si se trata de una modelización más detallada.

La resolución empleada en el modelo, celdas de $10 \mathrm{~km}$ de lado, suaviza los detalles, por lo que los resultados deben interpretarse como grandes tendencias macroclimáticas que rigen la distribución de las formaciones estudiadas, sin que se tenga capacidad para dibujar con precisión los límites geográficos. Las variaciones microclimáticas, $\tan$ frecuentes en el abrupto relieve ibérico, 
resultan imposibles de detectar con la resolución de este modelo.

Las distribuciones de partida son consecuencia de varios factores: históricos, climáticos, autoecológicos, antrópicos, etc., que no permiten conocer $a$ priori si la distribución que está siendo utilizada para modelizar se aproxima a la situación primitiva.

Además, las distribuciones utilizadas son incompletas, especialmente en el caso del alcornoque, que presenta un área de distribución extensa portuguesa, y que no es tenida en cuenta en este trabajo. La técnica predicitiva utilizada es especialmente sensible a estas carencias, ya que para producir la respuesta o predicción lo hace "supervisadamente" es decir, a partir de los datos de presencia con los que se entrena. En la Península, esta especie se presenta en un ambiente más o menos montano, en el área española, y en otro más basal en Portugal. En estos casos, para entrenar correctamente la red se deberían tomar datos de presencia de la especie bajo todos sus comportamientos, como ocurre en el caso de la encina.

\section{LA POTENCIALIDAD DE LOS ENCINARES}

El área estimada para los encinares españoles mediante redes neuronales confirma en lo esencial anteriores interpretaciones sobre la potencialidad de estos bosques (Font Quer, 1954; RivasMartínez, 1987). Sin embargo, la comparación con el mapa de series de vegetación potencial pone de manifiesto ciertas discrepancias en las áreas termomediterráneas y submediterráneas. Los encinares termomediterráneos de la alianza Querco rotundifoliae-Oleion sylvestris y algunos mesomediterráneos inferiores, de acuerdo con el modelo bioclimático propuesto, no resultan potenciales, ni en el valle del Guadalquivir ni en el litoral levantino. Se trata de un territorio conflictivo que ha sido interpretado de forma contrapuesta con anterioridad. Los autores catalanes (Bolòs, 1967; Folch, 1981) consideran los encinares levantinos como una formación finícola. Los interpretan como una variante termófila de los alsinares catalanes que habrían perdido buena parte de sus características. En esas zonas proponen como formaciones clímax bosques esclerófilos dominados por acebuches y lentiscos o comunidades arbustivas de tipo "maquia" caracterizadas por la coscoja y el lentisco (QuercoLentiscetum). Estiman que el encinar, "que ocuparía en el paisaje natural una extensión difícil de concretar", solo aparecería "en algunos rincones frescos y en suelos húmedos aluviales" (Bolòs, 1987). Por el contrario, la escuela fitosociológica
(Costa et al., 1982; Rivas-Martínez, 1987), considera el carrascal termófilo levantino como la clímax de gran parte del piso termomediterráneo. Aunque cabe pensar en una gran expansión de las garrigas y pinares como consecuencia de la acción antrópica, especialmente del aumento de los incendios, el modelo propuesto en este trabajo resalta la escasa importancia que tendría la encina en estos territorios. De manera similar, en el valle del Guadalquivir, aunque con una pequeña probabilidad de presencia de encina, habría que pensar en la existencia de formaciones mixtas termófilas de coscoja, lentisco, labiérnago, palmito, muy semejantes a las que se encuentran en el norte de África (Costa Tenorio et al., 1997).

En las zonas submediterráneas, entorno a los macizos montañosos y en las parameras de la submeseta norte, el área propuesta por el modelo es más amplia que las series de vegetación potencial, extendiéndose por territorios dominados por melojares y quejigares, e incluso algunos tipos de pinares o sabinares. En estas formaciones las encinas aparecen con frecuencia subordinadas a la especie principal por lo que parece que el modelo responde más bien a la ecología de la especie que a la del bosque. Como ya hemos señalado anteriormente, al tratarse de un modelo climático, no se tienen en cuenta otros factores, como la competencia con otras especies mejor adaptadas y que probablemente serían dominantes en estos territorios. Sin embargo, esto no excluye que en determinadas situaciones, suelos rocosos, exposiciones de solana, etc., la encina cobre importancia en estas áreas.

\section{LA POTENCIALIDAD DE LOS ALCORNOCALES}

Como hipótesis de trabajo resultaba esperable al modelizar la distribución de los alcornocales el obtener un área más amplia y continua que la distribución actual. No en vano decía Font Quer en 1954: "el área del alcornoque ha debido ser más extensa que la actual en la península Ibérica, se puede juzgar de ello por los restos dispersos de esta especie que observamos en la mayor parte de la Península". Aparecen localidades aisladas en La Liébana, al pie de los Picos de Europa, en múltiples enclaves de la costa cantábrica y galaica (Rias Baixas, península de Morrazo), en Euskadi, en las sierras de Bozóo y Besantes en la cuenca superior del Ebro, en el Pardo, Hoyo de Manzanares o la sierra de la Cabrera en Madrid, en la sierra de la Virgen al sur del Moncayo, en varios puntos de las cordilleras litorales mediterráneas aprovechando enclaves con rodenos, etc. 
Muchos otros autores suscriben esta misma opinión e interpretan esas pequeñas poblaciones dispersas de alcornoques que aparecen por todo el territorio peninsular, incluso en la costa cantábrica, como restos de una primitiva área continua o al menos mucho más amplia (Ceballos y Ruiz de la Torre, 1971; Montoya, 1988; Costa Tenorio et al., 1997; Carrión et al., 2000).

Aunque no se partía de todas las manifestaciones puntuales del alcornoque, al no estar cartografiadas en el mapa forestal de Ceballos (1966) que se utilizó para entrenar la red, parecía lógico esperar que el territorio óptimo para la especie ocupase una superficie mucho mayor y más continua, sobre todo en el cuadrante suroccidental ibérico que es donde mejor conservados y más abundantes son estos bosques y mayor la competencia con la encina. Dicha zona era considerada territorio potencial de alcornocales por el mapa de vegetación del Consejo de Europa (1979) o por Montoya (1988) aunque otros autores (Lautensach, 1967; Costa Tenorio et al., 1997) hacían más hincapié en la importancia de las dehesas mixtas en las que la potencialidad y el origen de ambas especies no parecía estar muy claro.

Siempre surge la duda cuando se modeliza la distribución de cualquier especie afectada por el proceso de "frutalización" de los paisajes mediterráneos. Todas las especies interesantes para el hombre han debido sufrir expansiones y retracciones escalonadas en el tiempo, y no necesariamente simultáneas que han modelado o perfilado sus áreas. En el caso de la encina y el alcornoque probablemente se viera en primer lugar favorecida la primera por su bellota comestible, que fue la base de la alimentación de las poblaciones paleolíticas, paralelamente a lo que ocurrió en el neolítico o en época romana con el castaño. Después, ya en periodo histórico, pudo propiciarse la extensión del alcornoque por la utilidad del corcho en la fabricación de envases, colmenas o tapones, y también por su bellota de maduración escalonada respecto a la de la encina que asegura un aprovechamiento más prolongado de la montanera en la dehesa. Estas fluctuaciones pueden haberse extendido hasta época reciente como reflejo de los mejores momentos o las crisis de la industria del corcho. Consecuencia de ello es que las distribuciones de partida están fuertemente influidas por las actividades antropozoógenas y que sufren incluso importantes cambios en los últimos años (Maldonado et al., 2002).

Como se ha comentado anteriormente, los resultados de la aplicación del modelo han resultado contundentes y muy diferentes de la hipótesis de partida. El área óptima para los alcornocales ha resultado bastante similar a la distribución real e incluso algo más restringida espacialmente (fig. 4). Completamente diferente de lo que ha ocurrido con la encina y con bastantes coincidencias con la propuesta de series de vegetación de Rivas-Martínez (1987). En relación con ésta, tan solo aparece una discrepancia relevante respecto a la serie OleoQuerceto suberis, alcornocales termófilos de la alianza Querco rotundifoliae-Oleion sylvestris (especialmente la faciación gaditano-onubense sobre arenales con Halimium halimifolium), que no resulta potencial según el modelo. Los territorios termomediterráneos litorales en los que la interpretación de la "clímax" ha dado lugar a numerosas controversias, porque están muy transformados desde antiguo y quedan pocos restos de vegetación natural, no resultan climáticamente óptimos, según la red neuronal aplicada, ni para la encina, ni para el alcornoque. En consecuencia el modelo conduce a defender la potencialidad en las llanuras litorales de comunidades de tipo "maquia o garriga", relacionables con la alianza Oleo-Ceratonion (Rivas Goday, 1966). Estas formaciones, que en ocasiones pueden llegar a alcanzar la talla y estructura propias de un verdadero bosque mixto mediterráneo termófilo (Costa Tenorio et al., 1997), estarían constituidas por un elevado número de especies. Entre ellas destacan árboles, arbustos o arbolillos como el algarrobo, el acebuche, el lentisco, la coscoja, el palmito, los espinos, madroños, labiérnagos o aladiernos. La encina estaría prácticamente ausente y el alcornoque podría aparecer, pero solo en los suelos ácidos y arenosos. Para la interpretación del valle del Guadalquivir, carente casi por completo de testimonios de vegetación natural, puede buscarse inspiración en algunas cuencas simétricas en el Norte de África como las del Sebou o el Loukhos, en la península Tingitana, que también tienen vertisoles arcillosos. Estas zonas todavía conservan retazos de estos lentiscares con acebuches, palmitos y coscojas, comunidades que fueron consideradas potenciales por Font Quer y Emberger en el mapa fitogeográfico de Marruecos (Emberger, 1939).

La red neuronal solo reconoce para los alcornocales un comportamiento montano en las alineaciones paleozoicas del suroeste peninsular y basal en el norte de Cataluña restringiendo de forma notable su nicho en España con respecto al de la encina.

Tradicionalmente, la distribución del alcornoque se ha explicado considerando el carácter acidófilo de la especie, por lo que sorprende que, partiendo exclusivamente de variables climáticas, el modelo haya estimado un área tan similar a la actual. 


\section{Conclusiones}

La utilización de Redes Neuronales Artificiales se ha revelado como una herramienta muy útil para el análisis de la potencialidad de especies forestales.

El modelo confirma la potencialidad de la encina como taxon continental mesetario y el alcornoque como una especie termófila de media montaña húmeda.

La distribución potencial de los encinares muestra una gran continuidad mientras que la de los alcornocales presenta varios núcleos disyuntos. Estas pautas coinciden con la interpretación tradicionalmente asignada por los naturalistas a la potencialidad de estos bosques.

La potencialidad de las series termomediterráneas de encinares y alcornocales no parece sustentarse con el modelo climático desarrollado.

\section{Referencias}

Augustin, N. H., Cummins, R. P. \& French, D. D., 2001. Exploring vegetation dynamics using logistic regression and multinomial logit model. Journal of Applied Ecology, 38(5): 991-103.

BAUM, E. B. \& HaussLeR, D., 1989. What size net gives a valid generalization? Neural Computation, 1: 151-160.

Berry, P. M., Harrison, P. A., Dawson, T. P. \& Pearson, R., 2001. Integrated Impacts on Biodiversity. In: P. Loveland et al. (eds.). Regional Climate Impacts Studies in East Anglia and North West England, Final Report to MAFF, DETR and UKWIR. Soil Survey and Land Research Centre. Silsoe. 360 pp.

Bishop, C. M., 1995. Neural Networks for Pattern Recognition. Oxford University Press. Oxford. 482 pp.

Bolòs, O. DE, 1967. Comunidades vegetales de las comarcas próximas al litoral situadas entre los ríos Llobregat y Segura. Memorias de la Real Academia de Ciencias de Barcelona, 38(1): 1-269.

Bolòs, O. DE, 1987. Cataluña y la depresión del Ebro. In: M. Peinado y S. Rivas-Martínez (eds.). La vegetación de España. Colección Aula Abierta. Publicaciones de la Universidad de Alcalá de Henares. Alcalá de Henares: 309-347.

BRYAN, B. A. \& ADAMS, J. M., 2001. Quantitative and qualitative assessment of the accuracy of neurointerpolated mean annual precipitation and temperature maps for China. Cartography, 30(2): 1-14.

Carletta, J., 1996. Assesing agreement on classification tasks: the kappa statistic. Computational Linguistics, 22(2): 1-6.

Carrión, J. S., Parra, I., Navarro, C. \& Munuera, M., 2000. Past distribution and ecology of cork oak (Quercus suber) in the Iberian Peninsula: a pollen- analytical approach. Diversity and Distributions, 6: 29-44.

Ceballos, L., 1966. Mapa Forestal de España 1:400. 000. Ministerio de Agricultura. Madrid. 50 pp. + 20 mapas.

Ceballos, L. \& Ruiz de la Torre, J., 1971. Árboles y arbustos. E.T.S.I. Montes. Madrid. 512 pp.

Consejo DE Europa, 1979. Carte de la végétation des états membres du Conseil de L'Europe. 1:3.000.000. Comité Européen pour la sauvegarde de la nature et des ressources naturelles. Strasbourg. 97 pp. +3 mapas.

Costa Tenorio, M., Morla, C. \& Sainz Ollero, H. (eds.), 1997. Los bosques ibéricos: una interpretación geobotánica. Planeta. Barcelona. 572 pp.

Costa, M., Peris, J. B. \& Figuerola, R., 1982. Sobre los carrascales termomediterráneos valencianos. Lazaroa, 4: 37-52.

Díaz-Fernández, P., Jiménez, M. P., Catalán, G., Martín Albertos, S. \& GiL, L., 1995. Regiones de procedencia de Quercus suber L. ICONA. ETSI Montes. $49 \mathrm{pp}$.

Duckworth, J. C, Bunce, R. G. H. \& Malloch, A. J. C., 2000. Vegetation gradients in Atlantic Europe: the use of existing phytosociological data in preliminary investigations on the potential effects of climate change on British vegetation. Global Ecology and Biogeography, 9: 187-199.

EMBERGER, L., 1939. Carte phytogéographique du Maroc 1:1.500.000. In: Aperçu général sur la végétation du Maroc. Veröffentlichungen des Geobotanischen Institutes der ETH, Stiftung Rübel, Zurich, 14: 40157 (reimpresión en Emberger, 1971, Travaux de botanique et d'écologie. Masson. Paris. 520 pp.).

Evans, F., 1998. An Investigation into the use of maximum likelihood classifiers, decision trees, neural networks and conditional probabilistic networks for mapping and predicting salinity. MSc in Computer Science at the Curtin University of Technology. Australia. (http://www. cmis. csiro. au/Fiona. Evans/personal $/ \mathrm{msc} / \mathrm{html} /$ )

Folch, R., 1981. La vegetació dels Països Catalans. Ketres. Barcelona. 513 pp.

FonT Quer, P., 1954. La vegetación. En: M. Terán (ed.) Geografía de España y Portugal. Tomo II. Montaner y Simón. Barcelona: 145-271.

FRIEDMAN, J. H., 1991. Multivariate adaptative regression splines. Annals of Statistical Association, 76(367): 817-823.

GARDNER, M. W. \& Dorling, S. R., 1998. Artificial neural networks (the multilayer perceptron) - A review of applications in the atmospheric sciences. Atmospheric environment, 32(14/15): 2627-2636.

GoH, A. T. C., 1995. Back-propagation neural networks for modelling complex systems. Artificial Intelligence in Engineering, 9: 143-151. 
GONZÁLEZ BERNÁLDEZ, F., 1992. La frutalización del paisaje mediterráneo. En: Paisaje mediterráneo. Electa. Milán: 136-141.

Guisan, A. \& Zimmermann, N. E., 2000. Predictive habitat distribution models in ecology. Ecological Modelling, 135: 147-186.

Guisan, A., Edwards, T. C. \& Hastie, J. T., 2002. Generalized linear and generalizad additive models in studies of especies distribucion: setting the scene. Ecological Modelling, 157: 89-100.

Guisan, A., Weiss, S. B. \& Weiss, A. D., 1999. GLM versus CCA spatial modelling of plants species distribution. Plant Ecology, 143: 107-122.

Hilbert, D. W. \& Ostendorf, B., 2001. The utility of artificial neural networks for modelling the distribution of vegetation in past, present and future climates. Ecological Modelling, 146: 311-327.

Hirzel, A. H., Helfer, V. \& Metral, F., 2001. Assessing habitat-suitability models with a virtual species. Ecological Modelling, 145: 111-121.

Hirzel, A. H., Hausser, J., Chessel, D. \& Perrin, N., 2002. Ecological-niche factor analysis: how to compute habitat-suitability maps without absence data? Ecology, 83(7): 2027-2036.

Hornik, K., Stichcombe, M. \& White, H., 1989. Multilayer feedforward networks are universal approximators. Neural Networks, 2: 359-366.

Huntley, B., Berry, P. M., Cramer, W. \& Mcdonald, A. P., 1995. Modelling Present and Potential Future Ranges of Some European Higher Plants Using Climate Response Surfaces. Journal of Biogeography, 22(6): 967-1001.

IVErson, L. R., Prasad, A. \& Schwartz, M. W., 1999. Modeling potential future individual tree-species distributions in the eastern United States under a climate change scenario: a case study with Pinus virginiana. Ecological Modelling, 115: 77-93.

Iverson, L. R., Prasad, A. M., Hale B. J. \& Sutherland, E. K., 1998. Predicting abundance for 80 tree species following climate change in the eastern United States. Ecological Monographs, 68(4): 465-485.

KIRKBY, M., 2000. Limits to modelling in the Earth and environmenetal sciences. In: S. Openshaw \& T. J. Abrahart (eds.) Geocomputation. Taylor and Francis. London and New York: 365-377.

Lautensach, M., 1967. Geografía de España y Portugal. Vicens Vives. Barcelona. 814 pp.

Lecun, Y., Bottou, L., OrR, G. B. \& Müller, K.-R., 1998 "Efficient BackProp" in Neural Networks: Tricks of the trade. G. B. Orr \& K.-R. Müller (eds.). $44 \mathrm{pp}$.

Lee, J. H. W., Huang, Y., Dickman, M. \& JAYAWARDENA, A. W., 2003. Neural network modelling of coastal algal blooms. Ecological Modelling, 159: 179-201.
Lee, S., Cho, S. \& Wong, P. M., 1998. Rainfall Prediction Using Artificial Neural Networks. Journal of Geographic and Decision Analysis, 2(2): 233-242.

Lennon, J. J. \& Turner, R. G., 1995. Predicting the Spatial Distribution of Climate: Temperature in Great Britain. Journal of Animal Ecology, 64(3): 370-392.

LÓPEZ GonzÁLEZ, G., 2001. Los árboles y arbustos de la Península Ibérica e Islas Baleares. Mundi Prensa. Madrid. 1727 pp.

Maldonado, J., Benito, M., Sanchez de Dios, R. \& SAINZ, H., 2002. Evolución reciente de las áreas de los bosques esclerófilos ibéricos. Cambios deducidos a partir de la cartografía forestal. En: J. Charco (coord.). La regeneración natural del bosque mediterráneo en la Península Ibérica. ARBA-DGCONA. Madrid: 217-236.

Martín del Brío, B. \& SAnz Molina, A., 1997. Redes Neuronales y Sistemas Borrosos. Ra-Ma. Madrid. $387 \mathrm{pp}$.

MARTínez MartíneZ, J., 1997 . Modelización de la vegetación en la cuenca alta del río Narcea (Asturias-España). Memoria de Investigación. Biología de Organismos y Sistemas Forestales. Universidad de Oviedo. (http://www. etsimo. uniovi. es/ juantxo/Seminario/Seminario. html).

Miller, J. \& Franklin, J., 2002. Modeling the distribución of tour vegetación alliances using generalizad linear models and clasificacion trees with spatial dependence. Ecological Modelling, 157: 227-247.

MitásovÁ, H. \& MitAs, L., 1993. Interpolation by regularized spline with tension: I. Theory and implementation. Mathematical Geology, 25: 641-655.

Monserud, R. A., 1990. Methods for comparing Global Vegetation Maps. WP-90-40 IIASA. Luxembourg. $31 \mathrm{pp}$.

MontoyA, J. M., 1988. Los alcornocales. MAPA. Madrid. 267 pp.

Ninyerola, M., 2000. Modelització climàtica mitjançant tècniques SIG i la seva aplicació a l'anàlisi quantitativa de la distribució d'espècies vegetals a l'Espanya peninsular. Tesis doctoral. Universitat Autònoma de Barcelona. 202 pp. (inédita).

Parmesan, C., Ryrholm, N., Stefanescu, C., Hill, J. K., Thomas, C. D., Descimon, H., Huntley, B., Kaila, L., Kullberg, J., Tammaru, T.,. Tennent, W. J., Thomas J. A. \& Warren, M., 1999. Poleward shifts in geographical ranges of butterfly species associated with regional warming. Nature, 399: 579583.

Paruelo, J. M. \& Tomasel, F., 1997. Prediction of functional characteristic of ecosystems: a comparison of artificial neural networks and regression models. Ecological Modelling, 98: 173-186.

Pearson, R. G., Dawson, T. P., Berry, P. M., Harrison, P. A. \& LAMPINEN, R., 2000. Neural Network Modelling 
of the Potential Redistribution of Plant Species in Response to Climate Change. In: P. Sincak \& J. Vascak (eds.). Quo Vadis Computational Intelligence?- New trends and Approach in Computational Intelligence. Springer-Verlag. Heildelberg: 112-119.

Pérez-Llera, C., Fernández-Baizán, M. C., Feito J. L. \& GonZÁleZ Del VAlle, V., 2002. Local Short-Term Prediction of Wind Speed: A Neural Network Analysis. IEMSs The International Modelling and Software Society. Proceedings: 124-129.

RIVAS GoDAY, S., 1966. Mapa de vegetación en VVAA Memoria de los trabajos desarrollados en el Proyecto E25-CR 11 (EG-Sp-111). Instituto nacional de Investigaciones Agronómicas, $\mathrm{M}^{\mathrm{o}}$ de Agricultura. Madrid. 117 pp.

Rivas-Martínez, S., 1987. Mapa de Series de Vegetación de España. 1:400.000. ICONA. Madrid. 268 pp. + 29 mapas.

Robertson, M. P., Caithness, N. \& Villet, M. H., 2001. A PCA-based modelling technique for predicting environmental suitability for organism from presence records. Diversity and Distributions, 7: 15-27.
Sánchez Palomares, O., Sánchez Serrano, F. \& Carretero Carretero, P., 1999. Modelos y Cartografía de Estimaciones Climáticas Termopluviométricas para la España peninsular. MAPA. Madrid. $192 \mathrm{pp}$.

Seonne, J. \& Bustamante, J., 2001. Modelos predictivos de la distribución de especies: una revisión de sus limitaciones. Ecología, 15: 9-21.

Sykes, M. T., Colin Prentice, I. \& Cramer, W., 1996. A Bioclimatic Model for the Potential Distributions of North European Tree Species Under Present and Future Climates. Journal of Biogeography, 23(2): 203-233.

TERradAs, J., 2001. Ecología de la vegetación. De la ecofisiología de las plantas a la dinámica de comunidades y paisajes. Omega. Barcelona. 703 pp.

Vayssières, M. P., Plant, R. E. \& Allen-Díaz, B. H., 2000. Classification trees: An alternative non-parametric approach for predicting species distributions. Journal of Vegetation Science, 11: 679-694. 